\title{
(ThyroChek®): RESULTADOS PRELIMINARES
}

\section{Caro Editor,}

R esolvemos submeter para a sua apreciação os resultados preliminares que Rseguem, os quais foram apresentados há pouco, durante o $9^{\circ}$. Encontro Brasileiro de Tiróide (EBT), em Gramado. Como acreditamos que somos o único grupo no país que está testando no momento essa metodologia, julgamos interessante dar divulgação a todos, nesta sessão de Cartas ao Editor.

Abraços,

\section{Mário Vaisman}

Professor Adjunto de Medicina

Serviço de Endocrinologia

Hospital Clementino Fraga Filho

Universidade Federal do Rio de Janeiro

\section{AVALIĊÃO DA ACURÁCIA DE MÉTODO RÁPIDO DE DIAGNÓSTICO DO HIPOTIREOIDISMO (THYROCHEK®) - RESULTADOS PRELIMINARES}

\author{
Ines Donangelo, Sabrina Mendes Coelho, Patricia Teixeira, Vaneska \\ Spinelli, Alexandru Buescu ơ Mário Vaisman
}

Serviço de Endocrinologia do Hospital Universitário Clementino Fraga Filho - UFRJ

ThyroCheck, é um teste semi-quantitativo de TSH que se propõe fornecer em minutos o diagnóstico de hipotireoidismo primário. Advoga-se que esta informação seria útil no dia-a-dia do clínico/endocrinologista tanto em casos de pacientes com de suspeita de hipotireoidismo em screening ambulatorial como de hipotireoidismo severo (coma mixedematoso) onde a decisão rápida de tratamento pode ser crucial.

Trata-se de um ensaio imunocromatográfico realizado com uma gota espessa de sangue. Em 15 minutos obtém-se a informação semi-quantitativa do valor do TSH sérico: maior do que $5 \mathrm{mlU} / \mathrm{ml}$ (teste positivo) ou menor que $5 \mathrm{mlU} / \mathrm{ml}$ (teste negativo).

O laboratório responsável pela sua distribuição (Merck) informa que a sensibilidade do teste é de $98,7 \%$ e a especificidade de $98,9 \%$.

O objetivo da presente comunicação é analisar a acurácia deste teste, bem como verificar suas possíveis vantagens e limitações.

Ao longo de 10 meses (abril-1999 a fevereiro-2000) realizamos o ThyroChek® de 62 pacientes, 46 hipotireoideos (grupo teste) e 16 eutireoideos (grupo controle), dosando na mesma amostra de sangue o TSH sérico por quimioluminescência. 
Utilizamos o ThyroChek(®) de acordo com as orientações do fornecedor: (figura 1)

1. colocar uma gota espessa de sangue na janela $\mathbf{A}$

2. aguardar 90 segundos

3. aplicar 5 gotas de solução tampão na janela $\mathbf{B}$

4. em cerca de 15 minutos surge na janela $C$ a linha rosa controle; se uma segunda linha paralela surgir o teste é considerado positivo.

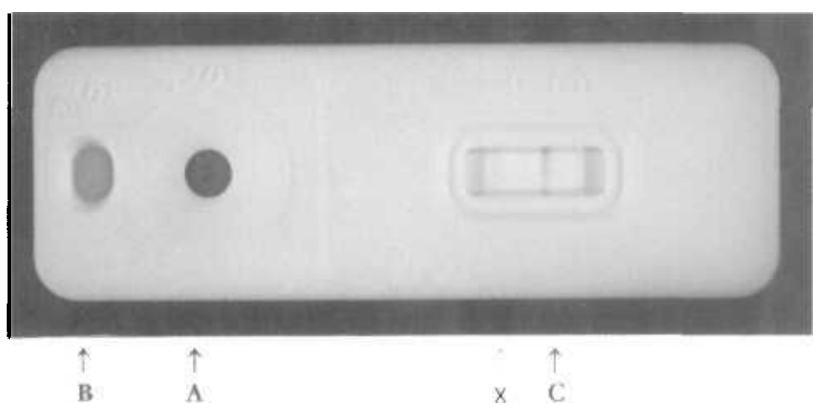

Figura 1 - Thyrochek $(B$ ) teste positivo (linha teste indicada pela seta $x$, paralela à linha controle)

A análise dos resultados foi feita por faixas de TSH sérico.

Tabela 1 - Grupo Controle - Eutiroideos

$\begin{array}{lc}\left.\text { ThyroChek }^{(}\right) & \text {Nivel Sérico TSH }<5 \mu \mathrm{lU} / \mathrm{ml} \\ & \\ \text { Positivo } & 3 \\ \text { Negativo } & 13 \\ \text { Total } & 16\end{array}$

Tabela 2 - Grupo Teste - Hipotiroideos

Thyrochek ${ }^{\circledR}$

Nivel Sérico de TSH (MIU/ml)

$5,1-10,0 \quad 10,1-15,0 \quad 15,1-20,0>20,1$ TOTAL

$\begin{array}{lccccc}\text { Positivo } & 5 & 6 & 4 & 21 & 36 \\ \text { Negativo } & 8 & 2 & 0 & 0 & 10 \\ \text { Total } & 13 & 8 & 4 & 21 & 46\end{array}$

A especificidade do ThyroChek( $($ foi de $81,2 \%$, com uma taxa de falso positivos de $18,8 \%$. A sensibilidade do teste para um TSH $>5,0 \mathrm{mlU} / \mathrm{ml}$ foi de $78,3 \%$, com uma taxa de falso negativos de $21,7 \%$.
Quando analisamos a sensibilidade de acordo com as faixas de TSH sérico, obscrvamos que a mesma aumenta claramente com valores mais elevados de TSH. Assim, a sensibilidade do teste para TSH entre 5,1 e $10 \mathrm{mlU} / \mathrm{ml}$ é de $38,4 \%$, para TSH entre 10,1 c $15 \mathrm{mlU} / \mathrm{ml}$, de $75,0 \%$ e para TSH acima de 15,1 é de $100 \%$.

Desta forma fica claro que o ThyroChek $®$ é um ótimo método para deteç̧ăo de TSH maior que 15,1 $\mathrm{mlU} / \mathrm{ml}$, regular para deteç̧ão de TSH entre $10,1 \mathrm{e}$ $15 \mathrm{mlU} / \mathrm{ml}$ e insatisfatório para deteç̧ão de TSH entre $5,1 \mathrm{e} 10 \mathrm{mlU} / \mathrm{ml}$.

Ao longo do estudo, notamos que detalhes téc nicos podem influenciar a leitura do resultado do teste pelo examinador:

A segunda linha que surge tornando o teste positivo foi bastante tênue em todos os casos, algumas vezes de difícil visualização, quando comparada com a intensidade da cor da linha controle;

Não observamos correlação entre a intensidade da cor da linha teste e o nível de 'TSH sérico;

A quantidade de sangue aplicada não foi rigorosamente igual entre os pacientes avaliados. Esta informação parece relevante se considerarmos os resultados de Dundas et al (1), que realizaram o ThyroChek® com sangue de pacientes hipotireoideos dividindo-os por faixa de TSH sérico $(4-6,6-10 \mathrm{e}>10$ $\mathrm{mUI} / 1)$ e por volume de sangue usado $(50,75,100 \mathrm{e}$ $125 \mathrm{ml}$ ). A sensibilidade foi de $100 \%$ para todas as faixas de TSH apenas quando se usou um volume de sangue de $125 \mathrm{ml}$ (cinco gotas). Nos volumes de $50 \mathrm{ml}$ e $75 \mathrm{ml}$ todos os testes foram falso negativos. Para volumes de $100 \mathrm{ml}$ a sensibilidade foi de $100 \%$ apenas em TSH $>10 \mathrm{mU} / 1$ e de $77,2 \%$ para valores entre 4 e 10 $\mathrm{mU} / \mathrm{l}$.

Notamos que alguns testes se tornavam positivos após os 15 minutos estabelecidos pelo fabricante para a leitura. No entanto, o minuto de "positivação" do teste não foi sistematicamente anotado.

Para melhor esclarecimento da acurácia do ThyroChck ${ }^{\circledR}$ e seu papel na prática clínica, mostra-se necessário o aperfciçoamento da técnica junto ao fabricante e avaliação de um número maior de pacientes.

\section{REFERÊNCIA}

1. Duntas LH, Koutras DA. Application of Thyrocheck in the assessment of the various degrees of hypothyroidism (letter). Thyroid 1999:9(8):847-8. 


\section{SINDROME DE TURNER: AVANÇOS E QUALIDADE DE VIDA}

\section{Senhor Editor,}

O impacto da aplicação de técnicas moleculares e de novas formas terapêuticas no diagnóstico e seguimento de pessoas portadoras da Síndrome de Turner, as coloca $\mathrm{em}$ situação privilegiada diante de outras síndromes genéticas, com a possibilidade de correção de grande parte dos estigmas, além de prevenir suas mais temidas complicações. Por outro lado, a grande oferta de ferramentas laboratoriais confunde o clínico, cioso de tratar e prevenir complicações com o menor número possível de procedimentos, de menor custo e menos agressivos. Evidencia-se assim, a necessidade do estabelecimento de normas mínimas essenciais a serem seguidas pelos profissionais envolvidos com o seu tratamento.

O Encontro de Especialistas em Síndrome de Turner: Avanços e Qualidade de Vida, levado a efeito no Centro de Extensão Universitária (rua Maestro Cardim, 370; Bela Vista, São Paulo), em novembro p.p., composto por especialistas agrupados de acordo com a respectiva área de atuação teve por objetivo gerar um documento de Consenso Nacional sobre a Síndrome. Os participantes e suas respectivas equipes foram:

\section{Equipe de Genética:}

Andrea Trevas Maciel-Guerra, Angela ViannaMorgante, Christine Heckel, Decio Brunoni, Isabel Melaragno, Janete Cerruti, Joyce Duffles Andrade, Maricilda Palandi de Mello e Silvia Souza da Costa.

\section{Equipe de Endocrinologia Clínica e Ginecológica: \\ Angela Maggio da Fonseca, Eduardo Leme} Alves da Mota, Elaine Maria Frade Costa, Ieda T.N. Verreschi, José Antonio Miguel Marcondes, Maria Teresa Matias Batista, Vilmon de Freitas, Paulo Augusto Junqueira e Marcia Marcelino de Souza.

\section{Equipe de Endocrinologia Pediátrica:}

Angela Spinola e Castro, Durval Damiani, Gil Guerra Júnior, Sofia Helena Valente de Lemos Marini e Marina Matsumoto.

\section{mo-Patologia \\ Equipe de Medicina Laboratorial e Anáto-}

Carlos Alberto Longui, Eduardo Vieira Neto, Francy dos Reys Patricio, José Carlos Lima, Judite R. Tavares Mendes, Marcia Marcelino de Souza, Rosana Delcelo e Tania Leme Martinez.

O documento gerado será colocado na "bomepage" da Sociedade Brasileira de Endocrinologia e Metabologia, ficando ao alcance de todos os interessados e recebendo sugestões para ampliação e aprimoramento do seu conteúdo. Após a disponibilização em rede, um documento acrescido das modificações sugeridas e acatadas, terá discussão ampla e redação definitiva em uma reunião que está agendada para o dia 5 de agosto próximo, já acordada entre os participantes do Encontro de Especialistas ocorrido em novembro p.p., no Centro de Extensão Universitária, São Paulo, SP.

Ao mesmo tempo que agradeço o seu empenho na divulgação deste documento, agradeço também o encaminhamento das sugestões sobre o documento que possam vir através dos Arquivos Brasileiros de Endocrinologia e Metabologia, bem como sobre a programação desta reunião final que, seguindo os moldes do encontro inicial, contará com a presença de especialistas das diferentes áreas do conhecimento empenhados em acompanhar e melhorar a qualidade de vida das pessoas portadoras desta síndrome tão freqüente.

Atenciosamente,

\section{Ieda T.N. Verreschi}

Professora Adjunta,

Disciplina de Endocrinologia e Metabologia

Departamento de Medicina,

Universidade Federal de São Paulo - Escola Paulista de Medicina 